\title{
Downregulation of GATA1 drives impaired hematopoiesis in primary myelofibrosis
}

\author{
Laure Gilles, ${ }^{1,2,3}$ Ahmet Dirim Arslan, ${ }^{4}$ Christian Marinaccio, ${ }^{1}$ Qiang Jeremy Wen, ${ }^{1}$ Priyanka Arya, ${ }^{1}$ Maureen McNulty, ${ }^{1}$ \\ Qiong Yang, ${ }^{1}$ Jonathan C. Zhao, ${ }^{1}$ Katerina Konstantinoff, ${ }^{1}$ Terra Lasho, ${ }^{5}$ Animesh Pardanani, ${ }^{5}$ Brady Stein, ${ }^{1,4}$ Isabelle Plo, ${ }^{2,3}$ \\ Sriram Sundaravel, ${ }^{6}$ Amittha Wickrema, ${ }^{6}$ Annarita Migliaccio, ${ }^{7,8}$ Sandeep Gurbuxani, ${ }^{9}$ William Vainchenker, ${ }^{2,3}$ \\ Leonidas C. Platanias, ${ }^{1,4,10}$ Ayalew Tefferi, ${ }^{5}$ and John D. Crispino ${ }^{1,4}$
}

'Division of Hematology/Oncology, Department of Medicine, Northwestern University, Chicago, Illinois, USA. ${ }^{2}$ INSERM, UMR 1170, and 3Paris Saclay, INSERM UMR 1170, Gustave Roussy, Villejuif, France. ${ }^{4}$ Robert H. Lurie Comprehensive Cancer Center, Northwestern University, Chicago, Illinois, USA. ${ }^{5}$ Department of Medicine, Mayo Clinic, Rochester, Minnesota, USA. ${ }^{6}$ Department of Medicine, University of Chicago, Chicago, Illinois, USA. 'Division of Hematology-Oncology, Mt. Sinai School of Medicine, New York, New York, USA. ${ }^{8}$ Università di Bologna, Bologna, Italy. ${ }^{9}$ Section of Hematopathology, University of Chicago, Chicago, Illinois, USA. ${ }^{10}$ Division of Hematology-Oncology, Department of Medicine, Jesse Brown VA Medical Center, Chicago, Illinois, USA.

\begin{abstract}
Primary myelofibrosis (PMF) is a clonal hematologic malignancy characterized by BM fibrosis, extramedullary hematopoiesis, circulating $\mathrm{CD} 34^{+}$cells, splenomegaly, and a propensity to evolve to acute myeloid leukemia. Moreover, the spleen and BM of patients harbor atypical, clustered megakaryocytes, which contribute to the disease by secreting profibrotic cytokines. Here, we have revealed that megakaryocytes in PMF show impaired maturation that is associated with reduced GATA1 protein. In investigating the cause of CATA1 downregulation, our gene-expression study revealed the presence of the RPS14-deficient gene signature, which is associated with defective ribosomal protein function and linked to the erythroid lineage in $5 q$ deletion myelodysplastic syndrome. Surprisingly, reduced CATA1 expression and impaired differentiation were limited to megakaryocytes, consistent with a proproliferative effect of a GATA1 deficiency on this lineage. Importantly, expression of GATA1 effectively rescued maturation of PMF megakaryocytes. Together, these results suggest that ribosomal deficiency contributes to impaired megakaryopoiesis in myeloproliferative neoplasms.
\end{abstract}

\section{Introduction}

A hallmark of primary myelofibrosis (PMF) is the presence of increased numbers of clustered, atypical megakaryocytes with hypolobulated, bulbous nuclei and an abnormal nuclear/cytoplasmic ratio (1). These abnormalities are recapitulated in vitro; previous studies have demonstrated that PMF progenitors in culture give rise to increased numbers of megakaryocytes, which display delayed apoptosis, altered proplatelet production, and secretion of increased levels of TGF- $\beta$ and matrix metallopeptidase 9 (MMP-9) $(2,3)$. Several lines of evidence suggest that aberrant megakaryocytes directly contribute to PMF. First, megakaryocytes lie in close proximity to hematopoietic stem cells and influence their cell cycle through secretion of TGF- $\beta$ and CXCL4 $(4,5)$. Second, elevated levels of megakaryocytes promote BM fibrosis in vivo $(6,7)$. Third, small molecules that induce maturation of malignant megakaryocytes show strong antitumor activity in myeloproliferative neoplasm (MPN) models (8). Here, we report a detailed analysis of the megakaryocyte lineage in PMF and reveal that impaired production of the transcription factor GATA1, downstream of a ribosomal defect, is the key event that leads to impaired megakaryocyte maturation.

Authorship note: A.D. Arslan and C. Marinaccio contributed equally to this work. Conflict of interest: The authors have declared that no conflict of interest exists. Submitted: June 3, 2015; Accepted: December 30, 2016.

Reference information: / Clin Invest. 2017;127(4):1316-1320.

https://doi.org/10.1172/JCI82905.

\section{Results and Discussion}

We first cultured primary human $\mathrm{CD} 34^{+}$cells from the peripheral blood of PMF patients and healthy donors in the presence of $10 \mathrm{ng} / \mathrm{ml}$ thrombopoietin (THPO) to induce megakaryocytic differentiation. As previously reported $(2,3)$, we observed that PMF cultures expanded to a much greater extent than those of healthy individuals (Supplemental Figure 1A; supplemental material available online with this article; https://doi.org/10.1172/ JCI82905DS1). After 9 days in culture, we observed an 11-fold expansion of PMF cells as compared with a 3-fold expansion of healthy cells. Furthermore, CFU assays revealed that PMF progenitors exhibited a higher capacity to form colonies than healthy cells (Supplemental Figure 1B). We obtained 3 to 4 times as many myeloid and erythroid colonies from the PMF CD34 ${ }^{+}$cells as compared with the healthy controls. The number of megakaryocyte colonies was also increased (Supplemental Figure 1C). We further observed significant reductions in the percentage of the mature $\mathrm{CD} 41^{+} \mathrm{CD} 42^{+}$population accompanied by a strong diminution of the ploidy level (Supplemental Figure 1D). On average, a 6-fold reduction in the $\mathrm{CD} 41^{+} \mathrm{CD} 42^{+}$population and an 8 -fold decrease in cells with a DNA content of $8 \mathrm{~N}$ or more were seen. Cytospins confirmed an abnormal morphology of the PMF megakaryocytes (Supplemental Figure 2).

Given the essential role for GATA1 in megakaryopoiesis, we assayed its expression in human patients and mouse models of PMF. In agreement with a previous report (9), we observed that the majority of megakaryocytes in the BM of a PMF patient did 
A

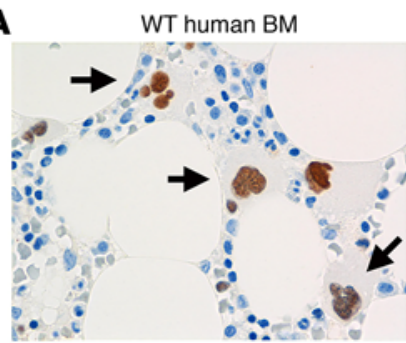

Human JAK2 mutant BM

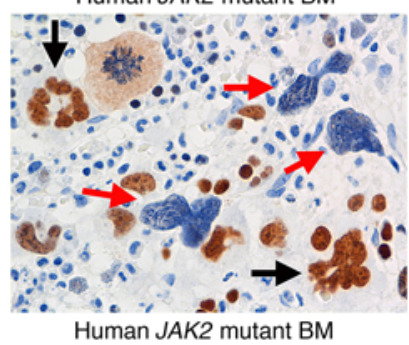

Human JAK2 mutant BM

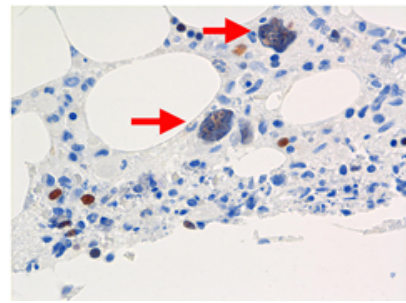

B

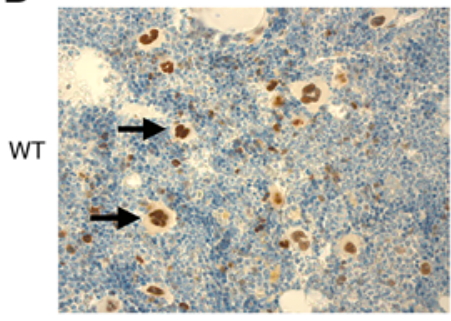

C

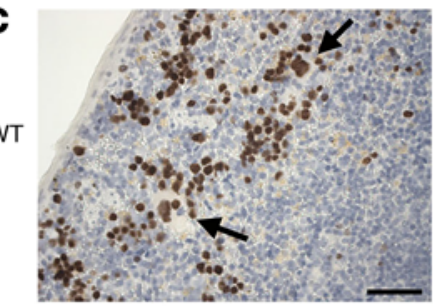

D

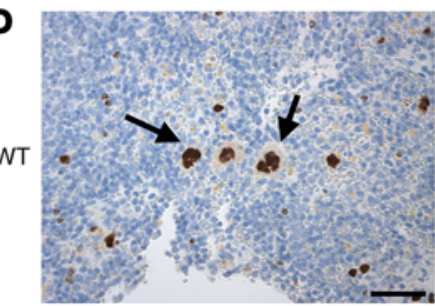

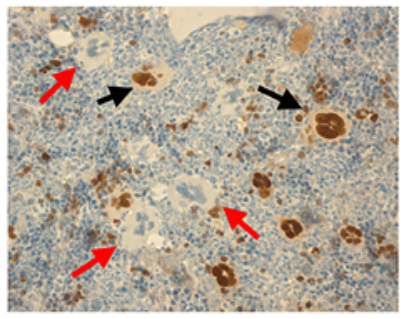

CALR

mutant

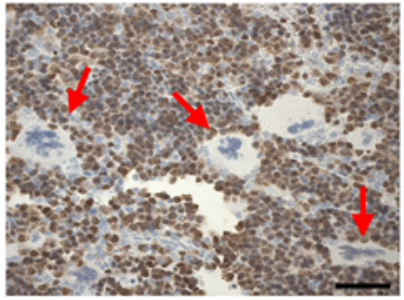

JAK2

mutant

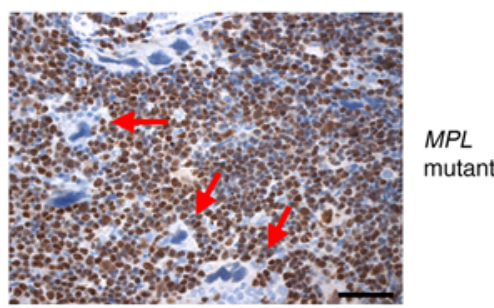

Figure 1. GATA1 protein expression is dysregulated in PMF and the mouse models of the MPNs. (A) Immunohistochemistry for GATA1 in sections of healthy BM and of BM from PMF patients with the JAK2V617F mutation. (B) Immunohistochemistry for GATA1 in sections of BM from mice transplanted with WT BM or with cells expressing the CALR type 1 mutant. (C and D) Immunohistochemistry for GATA1 in spleen sections from Jak2V617F Vav-Cre mice (C) or control littermates or spleens from mice transplanted with WT BM or with cells expressing MPLW515L (D). Red arrows, megakaryocytes that do not stain for GATA1; black arrows, megakaryocytes that express GATA1. Note the abnormal morphology of GATA1-deficient megakaryocytes in all models. Original magnification, $\times 500($ A); $\times 200$ (B-D).

not stain for GATA1 (Figure 1A). These GATA1-deficient megakaryocytes displayed the irregular morphology characteristic of PMF. We confirmed this downregulation of GATA1 in PMF patient megakaryocytes by Western blot (Supplemental Figure 3). Next, we performed immunohistochemistry on spleen and BM sections of mice that expressed calreticulin (CALR) type 1 mutant, either a V617F mutation in murine Jak2 (referred to hereafter as Jak2V617F) or a W515L mutation in human MPL (referred to hereafter as MPLW515L). In all cases, a preponderance of megakaryocytes did not stain for GATA1, and these cells exhibited an abnormal morphology (Figure 1, B-D). A similar reduction in GATA1 protein and morphologic abnormalities was observed in megakaryocytes from THPO-treated mice (10).

We then sorted $\mathrm{CD} 41^{+} \mathrm{CD} 42^{+}$cells derived from PMF patients and healthy donors (4 controls and PMF8-16, Supplemental Table 1), prepared mRNA, and performed a genome-wide geneexpression study. Analysis of the data revealed many changes in the PMF cells (Figure 2A). Most notably, several myeloid genes, whose expression is normally lost in mature megakaryocytes, were upregulated in comparison with controls. These included the enhancer binding protein $C E B P A$ and growth factor independent 1 (GFI1). Consistent with a regulatory circuit among GATA2, GFI1, and GFI1B, we also observed decreased expression of GATA2 and GFI1B (11). Of note, reducing CEBPA levels in PMF cells did not rescue the phenotype, while increased expression of GFI1 in healthy $\mathrm{CD} 34^{+}$cells did not recapitulate the defects seen in PMF (Supplemental Figures 4 and 5). Genes that were downregulated included several that are associated with megakaryocyte maturation, such as platelet factor 4 (PF4) (12). Interestingly, as previously reported (9), we observed that GATA1 mRNA was modestly elevated or unchanged relative to control megakaryocytes (Figure 2A). This result indicates that the loss of GATA1 protein is a posttranscriptional event.

Next, by Gene Set Enrichment Analysis (GSEA) (http:// software.broadinstitute.org/gsea/index.jsp), we identified several pathways that were altered in PMF megakaryocytes. These included hematopoietic cell lineage, AKT signaling, and MAP kinase pathways, the latter of which has already been reported (13) (Supplemental Figure 6A). To our surprise, one of the top scoring pathways was the ribosomal protein S14 (RPS14_DN.V1_UP) gene signature, which mimics $5 \mathrm{q}$ deletion myelodysplastic syndrome (MDS) (14) (Figure 2B). RPS14 is a component of the 40 S ribosomal subunit, and knockdown of this gene impairs $40 S$ ribosomal subunit formation (14). Of note, apart from the prominent erythroid defects, $5 \mathrm{q}$ deletion syndrome is characterized by the presence of abnormal hypolobulated megakaryocytes (15). In addition to the RPS14 gene signature, GSEA analysis revealed that 3 other ribosome-related pathways were suppressed in the PMF megakaryocytes. These include rRNA processing, ribosome biogenesis and assembly, and rRNA metabolic process (Supplemental Figure 6B). 
A

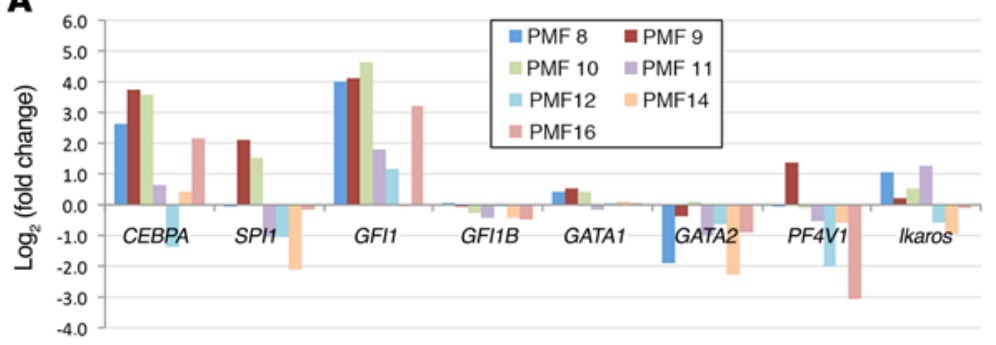

C
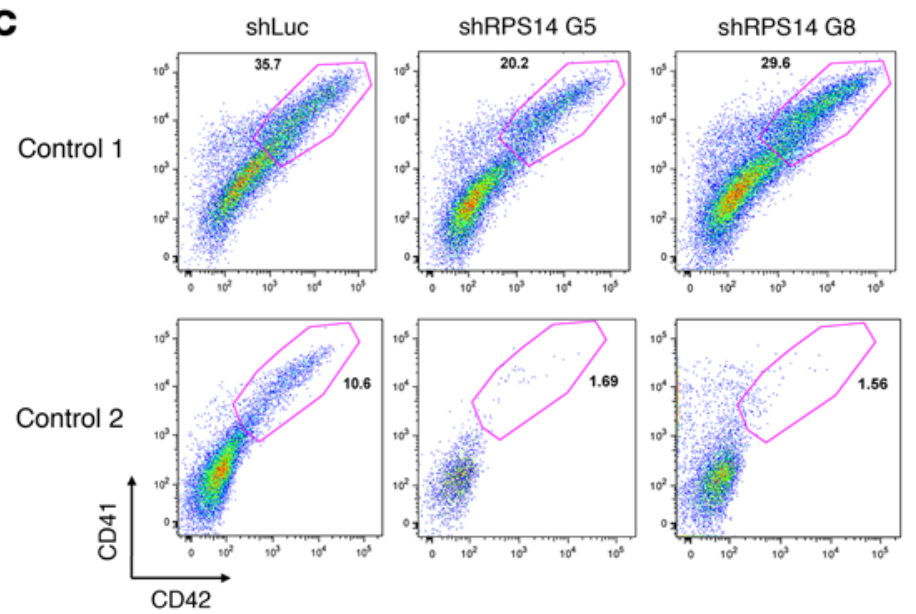

B

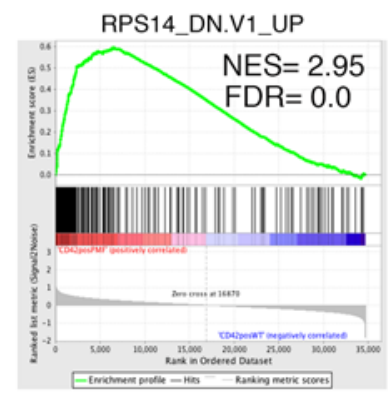

D
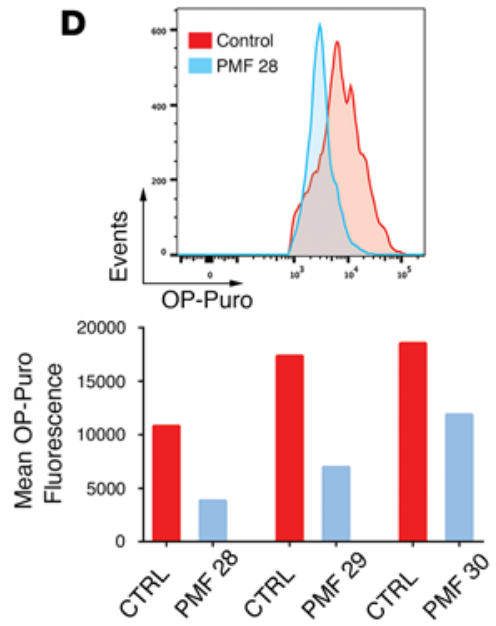

Figure 2. RPS14 is required for megakaryocyte maturation. (A) Fold change in expression of myeloid genes in megakaryocytes derived from PMF specimens or healthy control CD34+ cells. Results were derived from the gene-expression arrays. (B) GSEA analysis revealed that the RPS14_DN.V1_UP pathway (RPS14 gene signature) is significantly enriched. NES, normalized enrichment score; FDR, false discovery rate. (C) CD34+ cells were transduced with a control lentivirus or a lentivirus encoding either shRPS14 G5 or shRPS14 G8 and cultured under megakaryocytic differentiation conditions including $10 \mathrm{ng} / \mathrm{ml}$ THPO. Two representative experiments of 3 are shown. Megakaryocyte differentiation was reduced by $47 \% \pm 24 \%$ in the presence of shRPS14. $n=3$. $P=0.03$, Student's $t$ test. (D) Protein synthesis assay comparing PMF megakaryocytes with controls. Top panel: flow cytometry analysis of OP-PURO fluorescence in control versus PMF 28 CD42+ megakaryocytes. Bottom panel: mean OP-Puro fluorescence intensity for controls versus PMF samples.

Ribosome dysfunction is known to be associated with activation of the p53 pathway (16). The analysis of our microarray data revealed that the p53 pathway was indeed activated in PMF megakaryocytes (Supplemental Figure 7A). To confirm this signature, we performed quantitative reverse-transcriptase PCR (qRT-PCR) on control and PMF samples for 3 genes in the pathway: TP53, CDKN1A, and BAX. The results show increased expression of 2 p 53 targets in the PMF cells, confirming the activation of the pathway (Supplemental Figure 7B). We next knocked down RPS14 in the human JAK2 V617F mutant (JAK2V617F) SET2 megakaryocytic cell line and observed a nearly 4 -fold reduction of RPS14 expression, which was accompanied by an elevated expression of TP53, P21, and BAX (Supplemental Figure 7C).

To investigate whether the ribosomal gene changes and the reduction in GATA1 expression are restricted to megakaryocytes, we assayed the expression levels of a set of ribosome-associated genes in mononuclear cells and erythroid cells derived from PMF $\mathrm{CD} 34^{+}$cells. We did not observe significant changes in these genes in these 2 other lineages, suggesting that the ribosome deficiency is limited to megakaryocytes (Supplemental Figure 8). Similarly, we observed a reduction in ribosome-associated gene expression in megakaryocytes, but not erythroid cells, derived from CD $34^{+}$cells of polycythemia vera (PV) patients (Supplemental Figure 9 and
Supplemental Table 2). Moreover, differentiation of PV CD $34^{+}$cells revealed a striking impairment of megakaryopoiesis as assessed by CD41/CD42 staining and ploidy analysis (Supplemental Figure 10). This deficiency is consistent with the known abnormalities in the megakaryocyte lineage in PV; in this disease, megakaryocytes tend to form loose clusters, tend to be located close to boney trabeculae, and show marked pleomorphism with a mixture of different sizes. In addition, megakaryocytes with bulbous nuclei and hypercondensed chromatin can also be present, particularly with increasing BM fibrosis. We also note that there are morphologic abnormalities in megakaryocytes that fail to stain for GATA1 in mice with the Jak2V617F mutation (Figure 1C). Together, our data support a model in which enhanced JAK/STAT signaling leads to a selective impairment of the ribosome in megakaryocytes.

We next transduced human CD34 ${ }^{+}$cells with shRNAs targeting RPS14 (shRPS14G5 and shRPS14G8) or a control hairpin and then cultivated the cells under megakaryocyte differentiation conditions. Knockdown of RPS14 was confirmed by RT-PCR (Supplemental Figure 11). With knockdown, we observed a 1.5 -fold reduction in the $\mathrm{CD} 41^{+} \mathrm{CD} 42^{+}$population in cells transduced by shRPS14G5 and shRPS14G8 in comparison with control, although there was no change in the ploidy state of the cells (Figure $2 \mathrm{C}$ and 
A shLuc

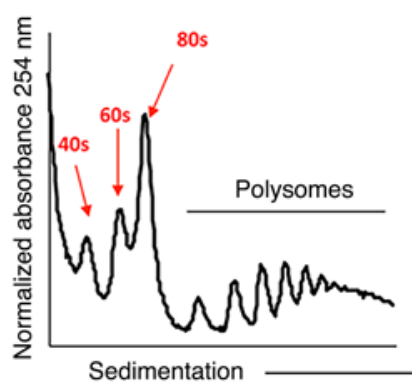

shRPS14 G5

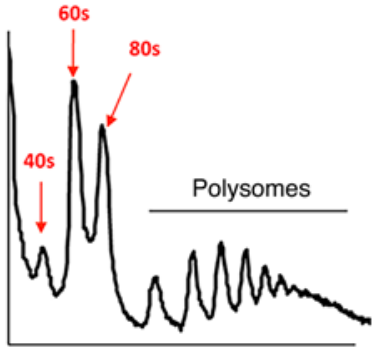

shRPS14 G8

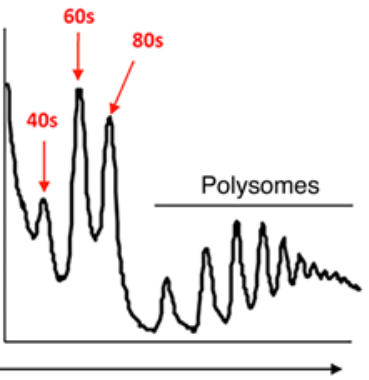

B

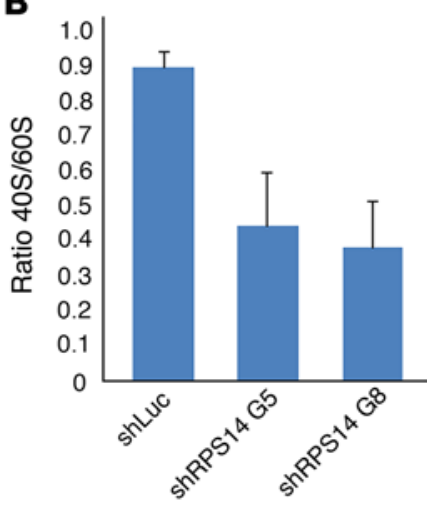

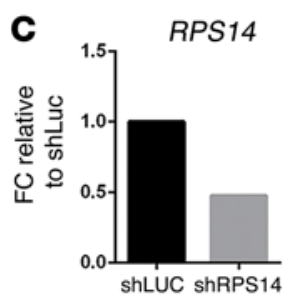
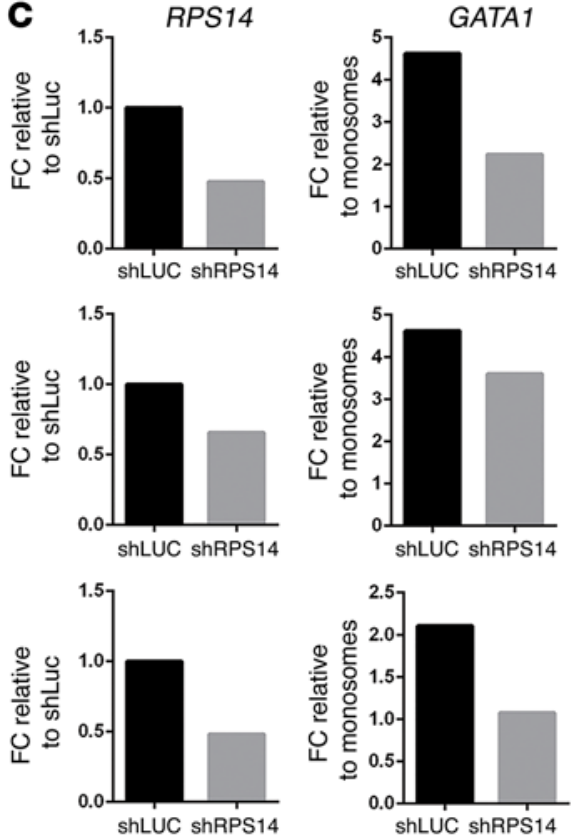

D

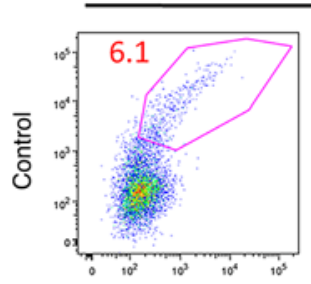

PMF 21
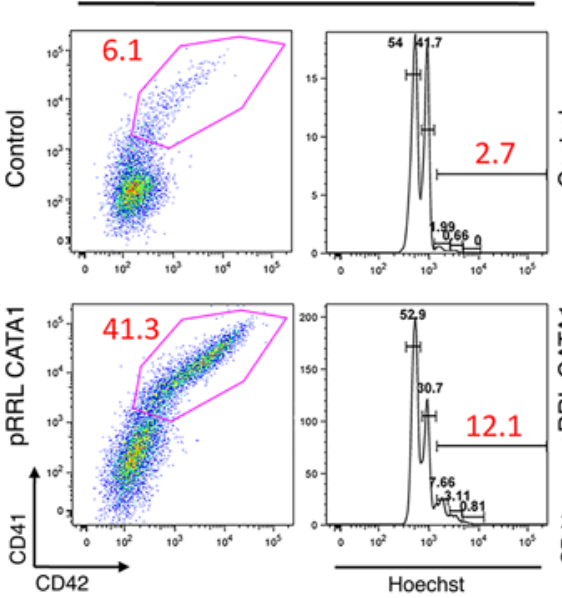

PMF 22

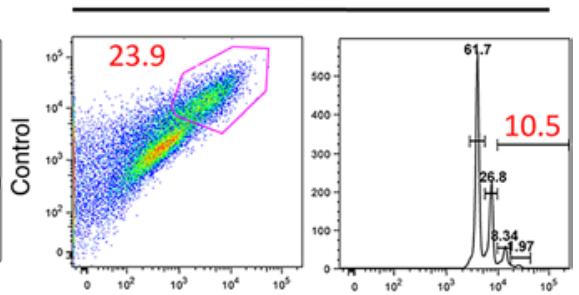

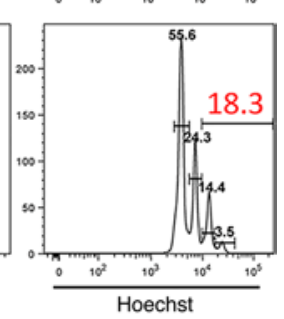

Figure 3. Restoration of GATA1 rescues the differentiation of PMF megakaryocytes. (A) Sucrose gradient polysome profiling showed a reduction in the 405 peak in SET2 cells overexpressing shRPS14 G5 or shRPS14 G8. The 40S, 60S, 80S, and ribosomal complexes are labeled. (B) Histogram showing the reduction in the ratio of 40S:60S subunits in the presence of 2 RPS14 shRNAs. Bar graph depicts mean \pm SD of 2 biological replicates. (C) qRT-PCR showing the fold change in RPS14 expression upon knockdown (left) and CATA1 mRNA abundance in polysomes compared with monosomes (right). Data were normalized to GAPDH. Three biological replicates are shown. (D) GATA1 was overexpressed in CD34 from PMF patients (PMF 21 and PMF 22). The numbers in red refer to the percentages of $\mathrm{CD} 41^{+} \mathrm{CD} 42^{+}$cells or the percentages of cells with DNA content of $8 \mathrm{~N}$ or more. Two representative experiments of 3 are shown. FC, fold change.

data not shown). This result reveals that a ribosome defect leads to aberrant megakaryopoiesis. To provide further evidence for a ribosome deficiency, we compared protein synthesis in megakaryocytes derived from PMF patients or healthy controls. We found that there was a significant reduction in protein synthesis in the PMF cells, consistent with a prominent ribosomal deficiency (Figure 2D and Supplemental Figure 12).

Together, our results are reminiscent of the recent discovery that ribosomal gene mutations in Diamond-Blackfan anemia (DBA) lead to reduced GATA1 translation $(17,18)$. Indeed, we observed that knockdown of RPS14 in SET2 cells led to a reduction in GATA1 protein (Supplemental Figure 13A). As expected, RPS14 mRNA was efficiently decreased, but in contrast, GATA1 mRNA was modestly elevated (Supplemental Figure 13B). This observation confirms that the RPS14 deficiency acts at the protein, but not
mRNA, level. We also discovered an increase in the levels of genes that are normally suppressed by GATA1, such as CEPBA, MYC, $M Y B, S P I 1$, and IKZF1 (Supplemental Figure 13C).

To investigate how GATA1 expression is affected by impaired ribosomes, we performed polysome profiling in SET2 cells after RPS14 knockdown. We saw that the RPS14 knockdown led to a reduction of the $40 \mathrm{~S}$ peak as well as the ratio of the $40 \mathrm{~S} / 60 \mathrm{~S}$ subunits (Figure 3, A and B). We then performed RT-PCR to quantify the abundance of GATA1 mRNA in actively translating ribosomes and observed a 1.3- to 2-fold reduction in GATA1 mRNA abundance in the polysomes of cells knocked down for RPS14 (Figure 3C). Since SET2 cells already have a JAK2 mutation and would be expected to have reduced GATA1 levels without RPS14 knockdown, we examined the effect of MPLW515L expression on GATA1 expression in a different megakaryocytic cell line, 6133 (19). We found that 6133 
cells with stable MPLW515L expression expressed significantly less GATA1 than the parental cells (Supplemental Figure 14). This result indicates that activation of JAK/STAT signaling is sufficient to lead to downregulation of GATA1 in megakaryocytes.

Finally, we created a lentiviral vector containing the human GATA1 cDNA and GFP and confirmed expression in transduced cells by RT-PCR (Supplemental Figure 15). Overexpression of GATA1 robustly improved differentiation of $\mathrm{CD} 4^{+}$cells from PMF patients (Figure 3D). The percentage of $\mathrm{CD} 41^{+} \mathrm{CD} 42^{+}$cells increased on average 3.5-fold upon GATA1 overexpression relative to GFP alone. Moreover, the percentage of cells with a ploidy of $8 \mathrm{~N}$ or more was increased 2.5-fold. As expected, we also observed restoration of GATA1 target gene expression (Supplemental Figure 16). Together, these data demonstrate that, although there are likely a number of proteins whose expression is reduced by the ribosome defect, the decline in GATA 1 is a critical driver of the phenotype.

Although lenalidomide has had a significant impact on the clinical course in MDS with $5 q$ deletion $(20,21)$, only modest results have been reported in PMF (22). As an alternative, compounds that induce megakaryocyte polyploidization of even GATA1-deficient cells (8) may provide therapeutic benefit by reducing the burden of abnormal megakaryocytes in patients with the MPNs. We note there are 2 limitations to the study. First, the mechanism by which MPN driver mutations cause a ribosomal defect in megakaryocytes is unclear. Second, the way in which a ribosomal defect in these cells leads to downregulation of translation of specific transcripts, such as GATA1, also requires further investigation. Nevertheless, our data reveal that the MPNs can be added to the list of hematopoietic diseases that are associated with ribosomal defects, including DBA, $5 q$ deletion syndrome, and Shwachman-Diamond anemia (23).

\section{Methods}

Detailed protocols are provided in Supplemental Methods. All original microarray data were deposited in the NCBI's Gene Expression Omnibus (GEO GSE84638).

Statistics. Data are shown as mean \pm SD. $P$ values were calculated using Student's $t$ test (2 tailed). $P \leq 0.05$ was considered to be significant.

Study approval. Peripheral blood was collected from patients who gave informed consent with approval of the Institutional Review Boards at the Mayo Clinic and Northwestern University. Animal studies were approved by the Northwestern University Institutional Animal Care and Use Committee.

\section{Author contributions}

LG, CM, ADA, QJW, PA, MM, QY, KK, and SS performed the experiments, analyzed the data, and contributed to writing the manuscript. JCZ performed informatics analysis and contributed to writing the manuscript. TL, AP, BS, IP, AW, and AT contributed patient samples, analyzed the data, and contributed to writing the manuscript. SG, AM, WV, and LCP analyzed the data and contributed to writing the manuscript. JDC directed the research and wrote the manuscript.

\section{Acknowledgments}

This work was supported by NIH grants R01HL112792 (to JDC); PO1 CA108671 (to AM); R01CA77816 and R01CA155566 (to LCP); and by the Samuel Waxman Cancer Research Foundation (to JDC). ADA was supported by NIH training grant T32CA070085. MM was supported by NIH training grant T32CA009560.

Address correspondence to: John Crispino, Northwestern University, 303 East Superior Street, 5-113, Chicago, Illinois 60611, USA. Phone: 312.503.1504; E-mail: j-crispino@northwestern.edu.
1. Thiele J, Kvasnicka HM, Müllauer L, BuxhoferAusch V, Gisslinger B, Gisslinger H. Essential thrombocythemia versus early primary myelofibrosis: a multicenter study to validate the WHO classification. Blood. 2011;117(21):5710-5718.

2. Ciurea SO, et al. Pivotal contributions of megakaryocytes to the biology of idiopathic myelofibrosis. Blood. 2007;110(3):986-993.

3. Balduini A, et al. In vitro megakaryocyte differentiation and proplatelet formation in $\mathrm{Ph}$-negative classical myeloproliferative neoplasms: distinct patterns in the different clinical phenotypes. PLOS ONE. 2011;6(6):e21015.

4. Bruns I, et al. Megakaryocytes regulate hematopoietic stem cell quiescence through CXCL4 secretion. Nat Med. 2014;20(11):1315-1320.

5. Zhao M, et al. Megakaryocytes maintain homeostatic quiescence and promote post-injury regeneration of hematopoietic stem cells. Nat Med. 2014;20(11):1321-1326.

6. Villeval JL, et al. High thrombopoietin production by hematopoietic cells induces a fatal myeloproliferative syndrome in mice. Blood. 1997;90(11):4369-4383.

7. Vannucchi AM, et al. Development of myelofibrosis in mice genetically impaired for GATA-1 expression (GATA-1(low) mice). Blood. 2002;100(4):1123-1132.

8. Wen QJ, et al. Targeting megakaryocytic- induced fibrosis in myeloproliferative neoplasms by AURKA inhibition. Nat Med. 2015;21(12):1473-1480.

9. Vannucchi AM, et al. Abnormalities of GATA-1 in megakaryocytes from patients with idiopathic myelofibrosis. Am J Pathol. 2005;167(3):849-858.

10. Vannucchi AM, et al. A pathobiologic pathway linking thrombopoietin, GATA-1, and TGF-beta1 in the development of myelofibrosis. Blood. 2005;105(9):3493-3501.

11. Moignard V, et al. Characterization of transcriptional networks in blood stem and progenitor cells using high-throughput single-cell gene expression analysis. Nat Cell Biol. 2013;15(4):363-372.

12. Lambert MP, Wang Y, Bdeir KH, Nguyen Y, Kowalska MA, Poncz M. Platelet factor 4 regulates megakaryopoiesis through low-density lipoprotein receptor-related protein 1 (LRP1) on megakaryocytes. Blood. 2009;114(11):2290-2298.

13. Desterke C, et al. FLT3-mediated p38-MAPK activation participates in the control of megakaryopoiesis in primary myelofibrosis. Cancer Res. 2011;71(8):2901-2915.

14. Ebert BL, et al. Identification of RPS14 as a 5qsyndrome gene by RNA interference screen. Nature. 2008;451(7176):335-339.

15. Mahmood T, Robinson WA, Hamstra RD, Wallner SF. Macrocytic anemia, thrombocytosis and nonlobulated megakaryocytes: the 5q-syndrome, a distinct entity. Am J Med.1979;66(6):946-950

16. Narla A, Ebert BL. Ribosomopathies: human disorders of ribosome dysfunction. Blood. 2010;115(16):3196-3205.

17. Ludwig LS, et al. Altered translation of GATA1 in Diamond-Blackfan anemia. Nat Med. 2014;20(7):748-753.

18. Bibikova E, et al. TNF-mediated inflammation represses GATA1 and activates p38 MAP kinase in RPS19-deficient hematopoietic progenitors. Blood. 2014;124(25):3791-3798.

19. Mercher T, et al. The OTT-MAL fusion oncogene activates RBPJ-mediated transcription and induces acute megakaryoblastic leukemia in a knockin mouse model. J Clin Invest. 2009;119(4):852-864.

20. List A, et al. Lenalidomide in the myelodysplastic syndrome with chromosome $5 \mathrm{q}$ deletion. $N$ Engl J Med. 2006;355(14):1456-1465.

21. Oliva EN, et al. Biological activity of lenalidomide in myelodysplastic syndromes with del5q: results of gene expression profiling from a multicenter phase II study. Ann Hematol. 2013;92(1):25-32.

22. Mesa RA, et al. Lenalidomide and prednisone for myelofibrosis: Eastern Cooperative Oncology Group (ECOG) phase 2 trial E4903. Blood. 2010;116(22):4436-4438.

23. McCann KL, Baserga SJ. Genetics. Mysterious ribosomopathies. Science. 2013;341(6148):849-850. 